\title{
Full quantum treatment of charge dynamics in amorphous molecular semiconductors
}

\author{
Xander de Vries, ${ }^{1}$ Pascal Friederich, ${ }^{2}$ Wolfgang Wenzel, ${ }^{2}$ Reinder Coehoorn, ${ }^{1,3}$ and Peter A. Bobbert ${ }^{1}$ \\ ${ }^{1}$ Department of Applied Physics, Eindhoven University of Technology, P.O. Box 513, NL-5600 MB Eindhoven, Netherlands \\ ${ }^{2}$ Institute of Nanotechnology, Karlsruhe Institute of Technology, D-76344 Eggenstein-Leopoldshafen, Germany \\ ${ }^{3}$ Institute for Complex Molecular Systems, Eindhoven University of Technology, P.O. Box 513, NL-5600 MB Eindhoven, Netherlands
}

(Received 20 September 2017; published 12 February 2018)

\begin{abstract}
We present a treatment of charge dynamics in amorphous molecular semiconductors that accounts for the coupling of charges to all intramolecular phonon modes in a fully quantum mechanical way. Based on $a b$ initio calculations, we derive charge transfer rates that improve on the widely used semiclassical Marcus rate and obtain benchmark results for the mobility and energetic relaxation of electrons and holes in three semiconductors commonly applied in organic light-emitting diodes. Surprisingly, we find very similar results when using the simple Miller-Abrahams rate. We conclude that extracting the disorder strength from temperature-dependent charge transport studies is very possible but extracting the reorganization energy is not.
\end{abstract}

DOI: 10.1103/PhysRevB.97.075203

\section{INTRODUCTION}

Amorphous polymeric and molecular organic semiconductors are crucial compounds used in organic devices such as organic field-effect transistors (OFETs), organic photovoltaic (OPV) cells, and organic light-emitting diodes (OLEDs). The modeling of charge dynamics in these materials is essential for the further development of organic devices. Systematic modeling started with the work of Bässler in the 1990s [1]. It was assumed that due to the disorder the charge carriers are localized and hop nonadiabatically between localization sites by phonon-assisted tunneling, or "hopping." The early studies made use of the Miller-Abrahams (MA) hopping rate [2], $k_{\mathrm{MA}}=k_{0}$ if $\Delta E<0$ and $k_{\mathrm{MA}}=k_{0} \exp \left(-\Delta E / k_{\mathrm{B}} T\right)$ if $\Delta E>0$, where $\Delta E$ is the electronic energy change, $T$ is temperature, $k_{\mathrm{B}}$ is Boltzmann's constant, and $k_{0}$ is a prefactor proportional to the square of the electronic coupling $J$ between the sites, assumed to decay exponentially with intersite distance. The advantage of using the MA rate is its simplicity: no material-specific information is needed for describing the energy dependence of the rate. It was established that the strength of the energetic disorder, modeled by a Gaussian density of states (DOS), plays a central role for both the steadystate mobility and energetic relaxation of charge carriers [1]. The resulting model is known as the Gaussian disorder model (GDM). It was later realized that filling of the DOS by only a small fraction of carriers can already strongly increase the charge-carrier mobility [3-5], requiring an extension of the GDM [6].

Present-day commercial OLEDs make use of amorphous molecular organic semiconductors. The theoretical advantage of considering molecular instead of polymeric semiconductors is that the localization sites are clearly defined: they coincide with the molecules. Recently, several groups made progress in evaluating charge dynamics in these materials from explicit calculations of the morphology, the on-site energies, the intermolecular electronic couplings, and the molecular reorganization energy $\lambda$ [7-10]. The semiclassical Marcus hopping rate [11] was used,

$$
k_{\text {Marcus }}=\frac{2 \pi}{\hbar} \frac{J^{2}}{\sqrt{4 \pi \lambda k_{\mathrm{B}} T}} \exp \left[-\frac{(\Delta E+\lambda)^{2}}{4 \lambda k_{\mathrm{B}} T}\right] .
$$

In Marcus theory, the coupling of the phonon modes to the charge is treated classically, which means that Eq. (1) is valid only if the phonon energy $\hbar \omega_{i} \ll k_{\mathrm{B}} T$ for all modes $i$. Because of the $\pi$ conjugation, intramolecular modes involving carboncarbon bond vibrations couple strongly to the charges. These modes have typical energies in the range $0.1-0.2 \mathrm{eV}$, i.e., almost one order of magnitude larger than $k_{\mathrm{B}} T$ at room temperature, invalidating a semiclassical approach and the use of Eq. (1). It is of paramount importance to study the consequences of going beyond the semiclassical approximation by including the charge-phonon coupling fully quantum mechanically and to provide benchmark results for the charge dynamics that do not suffer from uncontrolled approximations. That is the goal of this paper.

While Eq. (1) predicts a vanishing conductivity in the limit $T \rightarrow 0$, a quantum treatment of the phonon modes, accounting for nuclear tunneling through a classically forbidden region of nuclear arrangements accompanying the charge transfer, explains the observed finite conductivity in this limit in chemically doped in-plane diodes and ferroelectric field-effect transistors of amorphous semiconducting polymers [12]. In a recent study of low-energy tails of external quantum efficiency (EQE) spectra of OPV cells of $\mathrm{C}_{60}$ mixed with different donor molecules, a failure of the application of Eq. (1) in describing the EQE tails was attributed to quantum-mechanical freeze-out of high-energy phonons [13]. These two examples point to the importance of considering the quantum character of phonons in studying charge dynamics. We note that the impact of a full quantum treatment of phonon modes on charge transport has been considered for organic crystals [14] but, as far as we know, has not yet been considered for amorphous molecular semiconductors, where the percolative nature of the charge transport [15] adds an additional important complexity. 
This paper is organized as follows. In Sec. II we provide the expression for the full quantum charge transfer rate that is the basis of this work, as derived from Fermi's golden rule. In Sec. III we apply this expression to calculate the transfer rates of electrons and holes in three molecular semiconductors employed in OLEDs based on ab initio calculations of all intermolecular phonon frequencies and reorganization energies for simulated morphologies of these semiconductors. In Sec. IV we calculate the resulting mobilities of electrons and holes as well as their energetic relaxation in these three materials. Section V contains a summary and conclusion. Derivations of the formulas, the values of the calculated intermolecular phonon frequencies and their corresponding reorganization energies, and various checks and comparisons can be found in the appendixes.

\section{FULL QUANTUM CHARGE TRANSFER RATE}

Our description involves a charge, electron or hole, that can reside at two weakly electronically coupled molecules $\alpha=$ 1,2 . The charge couples with a strength $g_{\alpha i}$ to phonon modes $i$ with frequency $\omega_{i}$. The Hamiltonian is

$$
\begin{aligned}
H= & \sum_{\alpha} E_{\alpha} c_{\alpha}^{\dagger} c_{\alpha}+\sum_{i} \hbar \omega_{i} b_{i}^{\dagger} b_{i} \\
& +\sum_{\alpha, i} g_{\alpha i} \hbar \omega_{i} c_{\alpha}^{\dagger} c_{\alpha}\left(b_{i}^{\dagger}+b_{i}\right)+J\left(c_{1}^{\dagger} c_{2}+c_{2}^{\dagger} c_{1}\right),
\end{aligned}
$$

where $c_{\alpha}^{\dagger}\left(c_{\alpha}\right)$ creates (annihilates) a charge on molecule $\alpha$ and $b_{i}^{\dagger}\left(b_{i}\right)$ creates (annihilates) a phonon in mode $i$. Since firstprinciples calculations have shown that $J<0.01 \mathrm{eV}$ in the molecular semiconductors studied here [16], we can treat the last term in Eq. (2) as a perturbation $H^{\prime}$, causing nonadiabatic charge transfer between the molecules. The first part of the Hamiltonian can be diagonalized by a polaron transformation [17]. Applying Fermi's golden rule with $H^{\prime}$ as the perturbation, we obtain the transfer rate from molecule 1 to 2 by summing over all possible transitions from thermally weighted initial states to final states, with different numbers of phonons in the various modes, and the charge residing on molecule 1 or 2 [17]:

$$
\begin{aligned}
k= & \frac{2 \pi}{\hbar} J^{2} \exp \left(-\frac{\Delta E}{2 k_{\mathrm{B}} T}\right) \exp \left[-\sum_{i} \frac{\lambda_{i}}{\hbar \omega_{i}} \frac{1+z_{i}}{1-z_{i}}\right] \\
& \times \sum_{m_{1}, m_{2}, m_{3}, \ldots} I_{m_{1}}\left[2\left(\frac{\lambda_{1}}{\hbar \omega_{1}}\right) \frac{\sqrt{z_{1}}}{1-z_{1}}\right] \\
& \times I_{m_{2}}\left[2\left(\frac{\lambda_{2}}{\hbar \omega_{2}}\right) \frac{\sqrt{z_{2}}}{1-z_{2}}\right] I_{m_{3}}\left[2\left(\frac{\lambda_{3}}{\hbar \omega_{3}}\right) \frac{\sqrt{z_{3}}}{1-z_{3}}\right] \ldots \\
& \times \delta\left(\Delta E+\sum_{i} m_{i} \hbar \omega_{i}\right),
\end{aligned}
$$

with $z_{i} \equiv \exp \left(-\hbar \omega_{i} / k_{\mathrm{B}} T\right)$ and $\lambda_{i} \equiv \hbar \omega_{i} \sum_{\alpha} g_{\alpha i}^{2}$ being the reorganization energy associated with mode $i$. The integers $m_{i}$ in Eq. (3) are the numbers of created (positive $m_{i}$ ) or

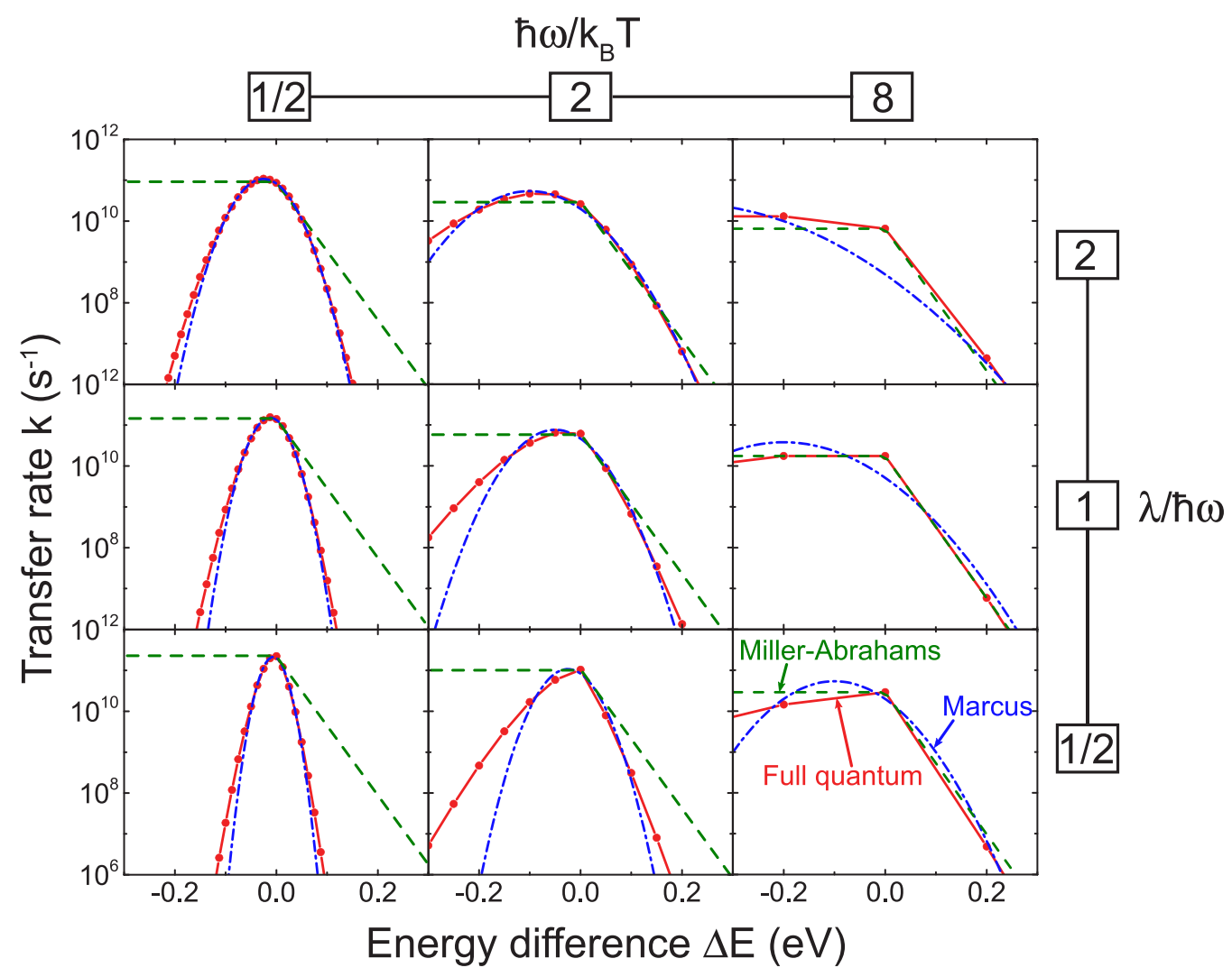

FIG. 1. Transfer rates at $T=300 \mathrm{~K}$ vs energy difference $\Delta E$ between two sites with electronic coupling $J=1 \mathrm{meV}$ and coupling to one phonon mode with varying frequency $\omega$ and reorganization energy $\lambda$. Red circles and solid lines: full quantum rate [prefactor of $\delta$ function in Eq. (3) divided by $\hbar \omega$ ]. Blue dash-dotted curves: Marcus rate. Dashed green lines: MA rate, scaled to reproduce the full quantum rate at $\Delta E=0$ 
annihilated (negative $m_{i}$ ) phonons in mode $i$ accompanying the transfer, and $I_{m_{i}}$ are modified Bessel functions. The $\delta$ function reflects energy conservation. A derivation of the rate equation (3) as well as an alternative expression that allows efficient numerical computation is given in Appendix A.

Using the properties of the modified Bessel functions, one can show that if for all modes $\hbar \omega_{i} \ll k_{\mathrm{B}} T$, Eq. (3) becomes equal to the Marcus rate equation (1) with $\lambda=\sum_{i} \lambda_{i}$. In the case of a continuous phonon spectrum, Eq. (3) becomes equal to a result given by Egger et al. [18]. The Marcus-LevichJortner formulation (MLJ) [19,20], which goes beyond the semiclassical approximation, assumes an initial state without phonons in the modes that are treated quantum mechanically. As a consequence, this theory does not satisfy detailed balance. As is easily checked, Eq. (3) does satisfy detailed balance. Appendix B shows how the MLJ rate is obtained from the full quantum rate equation (3) and contains a comparison of the two rates for the three semiconductors investigated in this work.

In Fig. 1 we show the $\Delta E$ dependence of the full quantum rate equation (3) at room temperature $(T=300 \mathrm{~K})$ for the case of one phonon mode for different values of its frequency and coupling strength. A typical value $J=1 \mathrm{meV}$ is chosen for the electronic coupling. For $\hbar \omega=k_{\mathrm{B}} T / 2$ (left column), the Marcus rate equation (1) is approximately recovered, as expected, while for $\hbar \omega=2 k_{\mathrm{B}} T$ and $8 k_{\mathrm{B}} T$ (middle and right columns) Eq. (1) is inaccurate. For $\hbar \omega=8 k_{\mathrm{B}} T$ and $\lambda=\hbar \omega$ or $2 \hbar \omega$ the full quantum rate is significantly larger than the Marcus rate around $\Delta E=0$, in accordance with the rate enhancement by nuclear tunneling [12]. Interestingly, the $\Delta E$ dependence of the full quantum rates for $\hbar \omega=8 k_{\mathrm{B}} T$ is close to that of the MA rate, in particular for $\lambda=\hbar \omega$. The reason is that for that case the prefactors of the $\delta$ function in Eq. (3) for $m=0$ and $m=1$ are approximately equal.

\section{ELECTRON AND HOLE TRANSFER RATES: APPLICATION TO $\alpha$-NPD, TCTA, and SPIRO-DPVBi}

We now turn to three specific molecular semiconductors employed in OLEDs, $\alpha$-NPD $\left[N, N^{\prime}-\operatorname{di}\left(1\right.\right.$-naphthyl)- $N, N^{\prime}-$ diphenyl-(1,1'-biphenyl)-4,4'-diamine] and TCTA [tris(4carbazoyl-9-ylphenyl)amine], both used as hole conductors and hosts for phosphorescent emitters, and spiro-DPVBi [2,2', 7, $7^{\prime}$-tetrakis(2,2-diphenylvinyl)spiro-9,9'-bifluorene $]$, used as an electron conductor and fluorescent emitter [21]. We recently studied charge transport in these materials using Marcus theory [16] on the basis of a simulated morphology and $a b$ initio calculations of the molecular on-site energies, reorganization energies, and electronic couplings. In Fig. 2(a) we display for these materials the cumulative reorganization energy $\lambda(\omega)=\sum_{\omega_{i}<\omega} \lambda_{i}$ for electrons and holes of 20 molecules randomly chosen from an amorphous sample as simulated in Ref. [16], taking into account all intramolecular modes. Appendix $\mathrm{C}$ explains how the frequencies $\omega_{i}$ and electron and hole reorganization energies $\lambda_{i}$ of these modes were obtained for the three materials and includes tables of their calculated values.

Clearly visible in Fig. 2(a) are the large contributions to $\lambda$ of modes involving $\mathrm{C}-\mathrm{C}$ bond vibrations in the range $0.1-0.2 \mathrm{eV}$. However, there are also important contributions from various dihedral rotations and other low-energy modes, particularly for

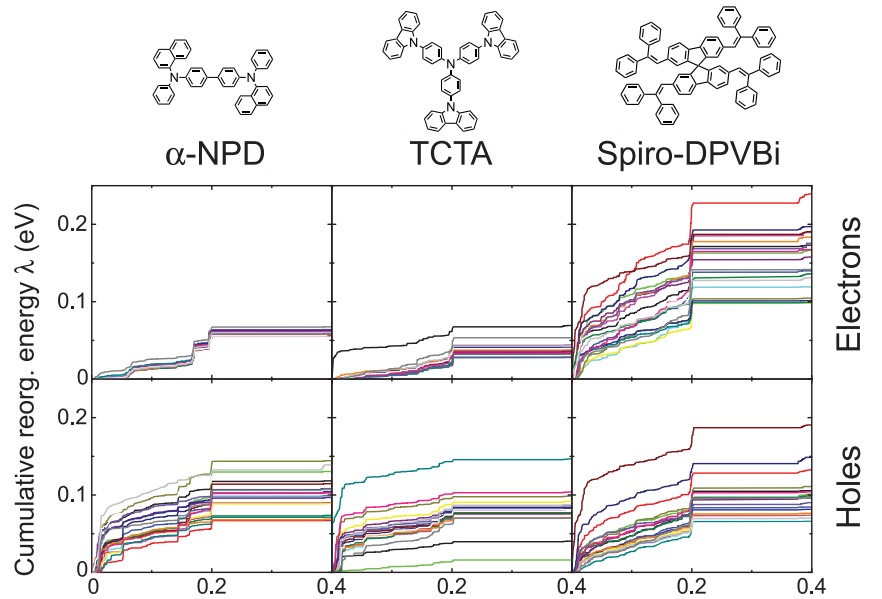

(a)

Phonon energy $\hbar \omega(\mathrm{eV})$

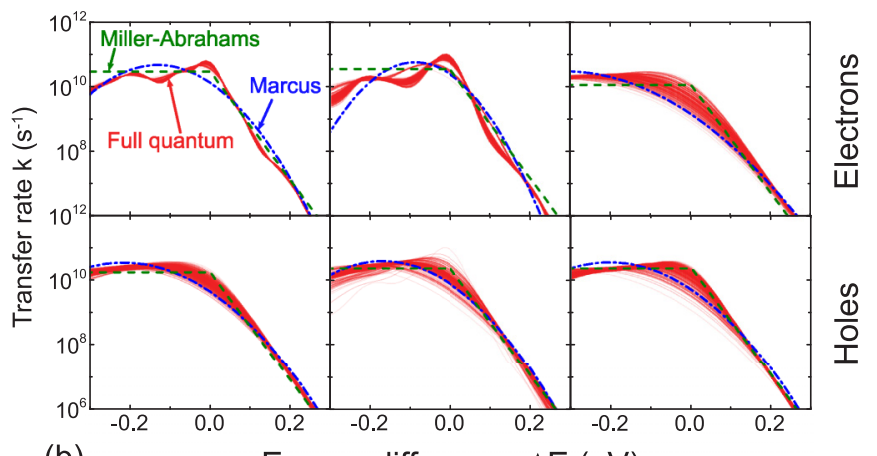

(b)

Energy difference $\Delta \mathrm{E}(\mathrm{eV})$

FIG. 2. (a) Cumulative reorganization energies for electrons and holes on 20 molecules randomly chosen from $\alpha$-NPD, TCTA, and spiro-DPVBi samples. (b) Energy dependence of transfer rates at $T=$ $300 \mathrm{~K}$ for $J=1 \mathrm{meV}$. Red curves: full quantum rates for all 400 combinations of molecules from (a), using $\lambda_{\mathrm{cl}}=0.01 \mathrm{eV}$. Blue dashdotted curves: Marcus rate using the average reorganization energy $\bar{\lambda}$ of the 20 molecules. Dashed green lines: MA rate with prefactor scaled to reproduce $\mu_{\text {full }}$ in Fig. 3(a) at $T=300 \mathrm{~K}$ and $F=0$.

electrons and holes in spiro-DPVBi and for holes in $\alpha$-NPD and TCTA, resulting in quasicontinuous spectra up to about $0.2 \mathrm{eV}$. Additionally, small contributions involving $\mathrm{C}-\mathrm{H}$ bond vibrations are visible just below $0.4 \mathrm{eV}$.

In the amorphous solid phase, apart from coupling to intramolecular modes, charges couple to intermolecular modes. Our present calculational framework does not allow us to evaluate this coupling. However, intermolecular modes will have low frequencies and can therefore be treated classically. Their effect can thus be accommodated by introducing a "classical" reorganization energy $\lambda_{\mathrm{cl}}$ and replacing the $\delta$ function in Eq. (3) by the right-hand side of Eq. (1) with $\lambda=\lambda_{\mathrm{cl}}$ (excluding the factor $2 \pi J^{2} / \hbar$ ), leading to a Gaussian broadening. We expect $\lambda_{\mathrm{cl}}$ to be considerably smaller than the intramolecular reorganization energies, but a small nonzero value is required in the charge dynamics calculations in the next section to allow for charge transfer at the various $\Delta E$ values occurring in the amorphous phase. In Appendix D we show for electrons in $\alpha$-NPD and TCTA (the two most critical cases) that in a broad range $0.001<\lambda_{\mathrm{cl}}<0.05 \mathrm{eV}$ the room-temperature mobility in the amorphous phase is practically independent of $\lambda_{\mathrm{cl}}$. In 
all results presented henceforth we take the computationally convenient value $\lambda_{\mathrm{cl}}=0.01 \mathrm{eV}$.

In Fig. 2(b) we show for this value and $J=1 \mathrm{meV}$ the $\Delta E$ dependence of the full quantum rates at room temperature for all 400 combinations of the molecules from Fig. 2(a), adding the phonon coupling spectra of two molecules to obtain the spectrum of their combination. The quasicontinuous spectra wash out features in the rates, except for electrons in $\alpha$-NPD and TCTA, for which the coupling to low-energy phonons is relatively small. For these two cases features related to the $0.2 \mathrm{eV}$ phonons are clearly visible. The results are reminiscent of those for $\hbar \omega=8 k_{\mathrm{B}} T$ at room temperature and $\lambda=\hbar \omega$ $\approx 0.2 \mathrm{eV}$ in Fig. 1. They can be understood as originating from a dominant mode of energy $\hbar \omega \approx 0.2 \mathrm{eV}$, with low-frequency modes "filling up" to a varying degree the regions in between integer multiples of this energy.

We also show in Fig. 2(b) the Marcus rates for the average reorganization energy $\bar{\lambda}$ of all combinations of molecules (the values are, respectively, $\bar{\lambda}=0.120,0.085$, and $0.299 \mathrm{eV}$ for electrons in the three materials and $\bar{\lambda}=0.212,0.184$, and $0.212 \mathrm{eV}$ for holes). In addition, we show MA rates with a prefactor tuned to reproduce the room-temperature zero-field mobilities (see the next section). The Marcus and the MA rates roughly follow the quantum rates in the displayed energy range, but differences up to an order of magnitude do occur. A comparison between the quantum rates and the MLJ rates is given in Fig. 4 in Appendix B, showing that these rates are inapplicable to modeling charge dynamics in amorphous molecular semiconductors.

We note that the scale of the phonon energies is about $0.1 \mathrm{eV}$, which is also the energy scale of the electron-phonon coupling since the electron-phonon coupling constants $g_{\alpha i}$ are of the order of unity for the important phonon modes. The on-site energy difference between two neighboring molecules is of the order of the disorder strength, which is also of the order of $0.1 \mathrm{eV}$. With values of $J$ that are, at most, $0.01 \mathrm{eV}$ [16], the energy scale of the electronic coupling is at least a factor of 10 smaller than that of the other terms in the Hamiltonian (A1). This justifies the use of perturbation theory in the electronic coupling in this work.

\section{MOBILITIES AND ENERGETIC RELAXATION OF ELECTRONS AND HOLES: APPLICATION TO $\alpha$-NPD, TCTA, AND SPIRO-DPVBi}

In evaluating the charge-carrier mobilities $\mu$ we follow the same procedure as in earlier work [16,22]. With a stochastic expansion method [23], simulated morphologies of the materials in relatively small simulation boxes are stochastically expanded to a collection of sites representing molecular centers of mass in boxes of size $100 \times 100 \times 100 \mathrm{~nm}^{3}$, large enough to allow reliable evaluation of $\mu$ by solving a master equation. A Gaussian DOS is taken for the site energies of electrons and holes, with standard deviations $\sigma$ equal to those of $a b$ initio calculations of the energies of the lowest unoccupied and highest occupied molecular orbitals (LUMO and HOMO) [24], respectively, of 1000 molecules in the simulated morphology (the values are, respectively, $\sigma=0.087,0.100$, and $0.156 \mathrm{eV}$ for electrons in the three materials and $\sigma=0.087,0.136$, and $0.122 \mathrm{eV}$ for holes [16]). We neglect spatial correlations in the site energies; they only weakly increase $\mu$ in the considered materials [16]. The electronic couplings $J$ between sites at a certain intersite distance are obtained by the stochastic method described in Ref. [22]. We randomly assign a label in the range 1-20 to each site and attribute one of the 400 transfer rates of Fig. 2(b) to each pair of electronically coupled sites (correcting the prefactor for the actual value of $J$ ) according to its label combination. We account for the presence of an electric field $F$ by adding an appropriate term to $\Delta E$.

Figure 3(a) shows the resulting $F$ dependence of the full quantum mobilities $\mu_{\text {full }}$ at $T=300$ and $200 \mathrm{~K}$, which we compare to $\mu_{\text {Marcus }}$ and $\mu_{\mathrm{MA}}$ obtained with the Marcus and MA rates. Since for spatially uncorrelated disorder the $F$ dependence of $\mu$ is approximately independent of the carrier concentration $c$ at not too high $F$ and $c$ [6], we take a convenient value $c=10^{-4}$ carriers per site, which is also realistic for OLEDs under operational conditions. The $F$-dependent $\mu$ at a different (not too high) $c$ can be obtained from the shown curves using the universal $c$ dependence of $\mu$ given in Ref. [15]. The mobilities $\mu_{\mathrm{MA}}$ were obtained by tuning the prefactor in the MA rate such that the mobilities $\mu_{\text {full }}$ at $T=300 \mathrm{~K}$ and $F=0$ are reproduced. The values of the MA prefactor correspond to, respectively, $k_{0} / J^{2}=2.92 \times 10^{16}$, $3.46 \times 10^{16}$, and $1.12 \times 10^{16} \mathrm{~s}^{-1} \mathrm{eV}^{-2}$ for electrons in the three materials and $k_{0} / J^{2}=1.79 \times 10^{16}, 2.41 \times 10^{16}$, and $2.15 \times 10^{16} \mathrm{~s}^{-1} \mathrm{eV}^{-2}$ for holes. It is gratifying to see that all these prefactors are of comparable magnitude.

Extremely large differences between $\mu_{\text {full }}, \mu_{\text {Marcus }}$, and $\mu_{\mathrm{MA}}$ do not occur in Fig. 3(a), which should be ascribed to the washing out of most of the differences in the rates in Fig. 2(b) by the energetic disorder. Still, significant differences between $\mu_{\text {full }}$ and $\mu_{\text {Marcus }}$ of up to a factor of $3 \mathrm{do}$, in some cases, occur. Quite strikingly, in those cases $\mu_{\text {MA }}$ yields a better description. By evaluating $\mu_{\text {Marcus }}$ using the separately calculated reorganization energies (see Tables I-III in Appendix C) instead of their average, leading to virtually the same results, we checked that the differences between $\mu_{\text {full }}$ and $\mu_{\text {Marcus }}$ are not caused by taking the average reorganization energy for the latter case.

Figure 3(b) shows the dependence of the $F=0$ mobilities on $\hat{\sigma}^{2} \equiv\left(\sigma / k_{\mathrm{B}} T\right)^{2}$ at a very low carrier concentration $c=$ $10^{-6}$, where the $c$ dependence of $\mu$ has become almost negligible. For comparison, we draw dashed lines corresponding to $\mu \propto \exp \left(-C \hat{\sigma}^{2}\right)$ with $C=0.4$, as approximately found in Ref. [16]. We conclude that this dependence is still obeyed rather well. Again, we observe that $\mu_{\mathrm{MA}}$ quite accurately describes $\mu_{\text {full }}$. At low $T$ we observe an enhancement of $\mu_{\text {full }}$ with respect to $\mu_{\text {Marcus }}$, amounting to a factor of about 3 for holes in $\alpha$-NPD at the lowest considered $T$, which can be attributed to nuclear tunneling. However, at room temperature (dotted line) this effect is, in all cases, unimportant.

We finally study energetic relaxation of carriers, which is claimed to play a crucial role in the functioning of bulk heterojunction OPV cells $[25,26]$. This relaxation has predominantly been studied using the MA rate $[1,25,26]$, and it is important to investigate the use of the full quantum rate. We evaluate energetic relaxation by solving a time-dependent master equation [27] in a $50 \times 50 \times 50 \mathrm{~nm}^{3}$ box with an equal occupational probability of all sites as the initial condition. Figure 3(c) shows for the three materials the time evolution of the average energy $\langle E\rangle$ of electrons and holes relaxing in 

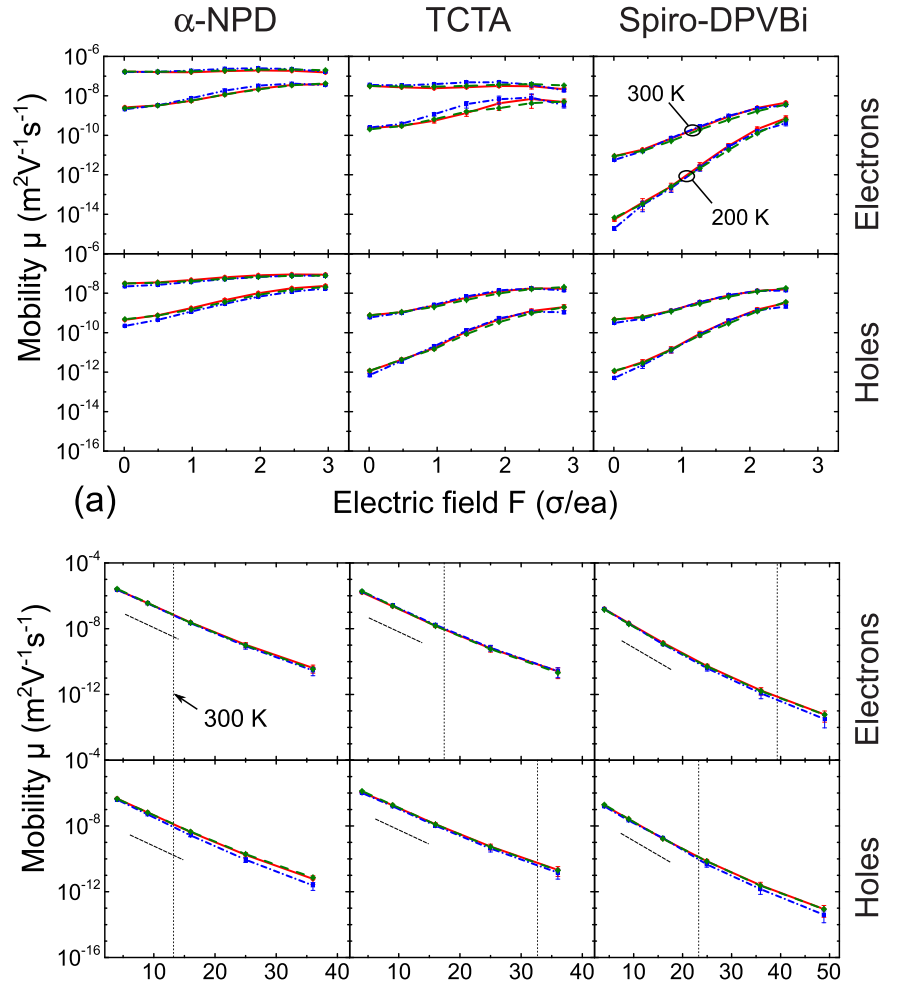

(b)

$\left(\sigma / k_{\mathrm{B}} \mathrm{T}\right)^{2}$

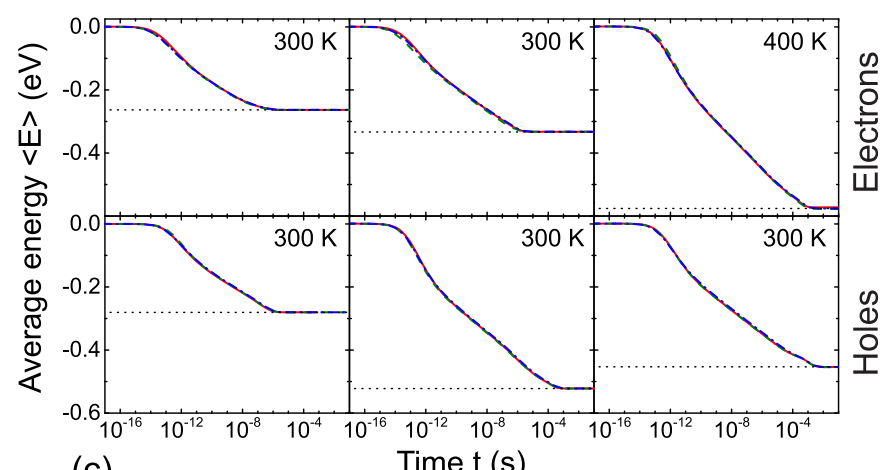

(c)

Time $t(s)$

FIG. 3. (a) Field-dependent mobilities at $T=300$ and $200 \mathrm{~K}$ and carrier concentration $c=10^{-4}$, where $e$ is the charge unit and $a$ is the average intersite distance $(1.01,1.05$, and $1.19 \mathrm{~nm}$, respectively, in the three materials [16]). The results are averages over ten disorder realizations. Red circles and solid lines: full quantum rates $\mu_{\text {full }}$. Blue squares and dash-dotted lines: Marcus rates $\mu_{\text {Marcus }}$. Green diamonds and dashed lines: MA rates $\mu_{\mathrm{MA}}$, scaled to reproduce $\mu_{\text {full }}$ at $T=300$ $\mathrm{K}$ and $F=0$. (b) Dependence of mobilities on $\hat{\sigma}^{2} \equiv\left(\sigma / k_{\mathrm{B}} T\right)^{2}$ for $c=10^{-6}$ and $F=0$. Dotted lines indicate $T=300 \mathrm{~K}$, and dashed lines indicate slopes corresponding to $\mu \propto \exp \left(-0.4 \hat{\sigma}^{2}\right)$. (c) Timedependent average energy, with respect to the center of the DOS, of carriers initially released at arbitrary sites $(T=400 \mathrm{~K}$ for electrons in spiro-DPVBi and $300 \mathrm{~K}$ for all other cases). Dotted lines indicate full relaxation.

the Gaussian DOS. The temperature is $T=300 \mathrm{~K}$, except for electrons in spiro-DPVBi, where for computational stability reasons related to the large value of $\sigma$ we took $T=400 \mathrm{~K}$. The results of using full quantum, Marcus, and MA rates are almost indistinguishable.

\section{SUMMARY AND CONCLUSION}

Summarizing, we have studied the charge dynamics in three molecular semiconductors used in OLEDs with a hopping rate that includes the coupling to all intramolecular phonon modes in a fully quantum mechanical way, improving on the widely used semiclassical Marcus rate. In some cases we found a significant modulation of the rate associated with $\mathrm{C}-\mathrm{C}$ bond vibrations. Benchmark results for the mobility and energetic relaxation of electrons and holes are obtained that are free from uncontrolled approximations. We find that nuclear tunneling can affect the mobility at low temperature. Surprisingly good results for the mobilities as well as the energetic relaxation of charge carriers are obtained with the simple and materialindependent Miller-Abrahams rate, which can be used as a computationally cost-effective alternative. We conclude that extracting the disorder strength from temperature-dependent charge transport studies, as initially proposed by Bässler [1], is very possible but extracting the reorganization energy is not.

\section{ACKNOWLEDGMENTS}

This research is part of the Horizon-2020 EU projects EXTMOS (Project No. 646176) and MOSTOPHOS (Project No. 646259) and of the Dutch-German project MODEOLED, supported on the Dutch side by the applied science division of NWO and the Technology Program of the Dutch Ministry of Economic Affairs (Project No. 12200) and on the German side by the "Deutsche Forschungsgemeinschaft" (Project No. WE1863/22-1).

\section{APPENDIX A: DERIVATION OF THE FULL QUANTUM CHARGE TRANSFER RATE}

In this appendix we give the derivation of the full quantum charge transfer rate equation (3). We start from the Hamiltonian equation (2), which we repeat here for convenience:

$$
\begin{aligned}
H= & \sum_{\alpha=1,2} E_{\alpha} c_{\alpha}^{\dagger} c_{\alpha}+\sum_{i} \hbar \omega_{i} b_{i}^{\dagger} b_{i} \\
& +\sum_{\alpha, i} g_{\alpha i} \hbar \omega_{i} c_{\alpha}^{\dagger} c_{\alpha}\left(b_{i}^{\dagger}+b_{i}\right)+J\left(c_{1}^{\dagger} c_{2}+c_{2}^{\dagger} c_{1}\right) .
\end{aligned}
$$

Part of the phonon modes couples to the charge on site 1 $\left(g_{1 i} \neq 0, g_{2 i}=0\right)$, and the other part couples to the charge on site $2\left(g_{1 i}=0, g_{2 i} \neq 0\right)$. We treat the last term, $H_{1}$, of the Hamiltonian, containing the electronic coupling $J$, as a perturbation causing transitions between the eigenstates of the first part, $H_{0}$, of the Hamiltonian (first three terms). We apply the Schrieffer-Wolff polaron transformation [17] to the Hamiltonian and define

$$
\begin{aligned}
& \bar{H}_{0} \equiv e^{S} H_{0} e^{-S}, \quad \bar{H}_{1} \equiv e^{S} H_{1} e^{-S}, \\
& S \equiv \sum_{\alpha, i} g_{\alpha i} c_{\alpha}^{\dagger} c_{\alpha}\left(b_{i}^{\dagger}-b_{i}\right) .
\end{aligned}
$$

By applying the Baker-Campbell-Hausdorff theorem,

$$
\begin{aligned}
e^{S} A e^{-S}= & A+\frac{1}{1 !}[S, A]+\frac{1}{2 !}[S,[S, A]] \\
& +\frac{1}{3 !}[S,[S,[S, A]]]+\cdots,
\end{aligned}
$$


and the (anti)commutation relations for the creation and annihilation operators in Eq. (A1), we obtain, after some algebra,

$$
\bar{H}_{0}=\sum_{\alpha} \bar{E}_{\alpha} \bar{c}_{\alpha}^{\dagger} \bar{c}_{\alpha}+\sum_{i} \hbar \omega_{i} \bar{b}_{i}^{\dagger} \bar{b}_{i}, \quad \bar{H}_{1}=J\left(\bar{c}_{1}^{\dagger} \bar{c}_{2} X_{1}^{\dagger} X_{2}+c_{2}^{\dagger} c_{1} X_{2}^{\dagger} X_{1}\right)
$$

with

$$
\bar{c}_{\alpha}^{\dagger}=e^{S} c_{\alpha}^{\dagger} e^{-S}, \quad \bar{c}_{\alpha}=e^{S} c_{\alpha} e^{-S}, \quad \bar{b}_{\alpha}^{\dagger}=e^{S} b_{\alpha}^{\dagger} e^{-S}, \quad \bar{b}_{\alpha}=e^{S} b_{\alpha} e^{-S}, \quad \bar{E}_{\alpha} \equiv E_{\alpha}-\lambda_{\alpha}, \quad \lambda_{\alpha} \equiv \sum_{i} g_{\alpha i}^{2} \hbar \omega_{i}, \quad X_{\alpha} \equiv e^{\sum_{i} g_{\alpha i}\left(\bar{b}_{i}^{\dagger}-\bar{b}_{i}\right)} .
$$

The creation and annihilation operators in $\bar{H}_{0}$ and $\bar{H}_{1}$ are transformed operators creating and annihilating polarons $\left(\bar{c}_{\alpha}^{\dagger}\right.$ and $\left.\bar{c}_{\alpha}\right)$ and "displaced" phonons $\left(\bar{b}_{\alpha}^{\dagger}\right.$ and $\left.\bar{b}_{\alpha}\right)$.

We consider initial states $|i\rangle$ where the polaron is at site 1 with $n_{i}$ phonons in mode $i$ and final states $|f\rangle$ and where the polaron is at site 2 with $n_{i}+m_{i}$ phonons in mode $i$. The matrix element for the transition $|i\rangle$ to $|f\rangle$ induced by the perturbation is

$$
\left\langle f\left|\bar{H}_{1}\right| i\right\rangle=J\left[\prod_{i, \text { site } 1}\left\langle n_{i}+m_{i} e^{g_{1 i}\left(\bar{b}_{i}^{\dagger}-\bar{b}_{i}\right)} \mid n_{i}\right\rangle\right]\left[\prod_{i, \text { site } 2}\left\langle n_{i}+m_{i} e^{-g_{2 i}\left(\bar{b}_{i}^{\dagger}-\bar{b}_{i}\right)} \mid n_{i}\right\rangle\right] .
$$

The phonon matrix elements in this expression can be easily evaluated:

$$
\begin{aligned}
\left\langle n_{i}+m_{i} e^{g_{1 i}\left(\bar{b}_{i}^{\dagger}-\bar{b}_{i}\right)} \mid n_{i}\right\rangle & =e^{-\frac{1}{2} g_{1 i}^{2}}\left(-g_{1 i}\right)^{m_{i}}\left[\frac{n_{i} !}{\left(n_{i}+m_{i}\right) !}\right]^{\frac{1}{2}} L_{n_{i}}^{m_{i}}\left(g_{1 i}^{2}\right), \\
\left\langle n_{i}+m_{i} e^{-g_{2 i}\left(\bar{b}_{i}^{\dagger}-\bar{b}_{i}\right)} \mid n_{i}\right\rangle & =e^{-\frac{1}{2} g_{2 i}^{2}}\left(g_{2 i}\right)^{m_{i}}\left[\frac{n_{i} !}{\left(n_{i}+m_{i}\right) !}\right]^{\frac{1}{2}} L_{n_{i}}^{m_{i}}\left(g_{2 i}^{2}\right),
\end{aligned}
$$

where $L_{n}^{m}(x)$ is an associated Laguerre polynomial. Inserting these matrix elements in Eq. (A6) and squaring leads to

$$
\left|\left\langle f\left|\bar{H}_{1}\right| i\right\rangle\right|^{2}=J^{2} \prod_{i} \exp \left[-\frac{\lambda_{i}}{\hbar \omega_{i}}\right]\left(\frac{\lambda_{i}}{\hbar \omega_{i}}\right)^{m_{i}} \frac{n_{i} !}{\left(n_{i}+m_{i}\right) !}\left[L_{n_{i}}^{m_{i}}\left(\frac{\lambda_{i}}{\hbar \omega_{i}}\right)\right]^{2},
$$

with $\lambda_{i} \equiv \hbar \omega_{i} \sum_{\alpha} g_{\alpha i}^{2}$ being the reorganization energy associated with mode $i$.

We now apply Fermi's golden rule to the transitions between all thermally populated initial states and all final states. The probability $p_{n_{i}}$ to have $n_{i}$ phonons in mode $i$ is

$$
p_{n_{i}}=\left(1-z_{i}\right) z_{i}^{n_{i}}, \quad z_{i} \equiv e^{-\hbar \omega_{i} / k_{\mathrm{B}} T} .
$$

This yields the following expression for the rate $k$ for a transition of the polaron from site 1 to 2 :

$$
\begin{aligned}
k & =\frac{2 \pi}{\hbar} \sum_{n_{1}, n_{2}, \ldots} p_{n_{1}} p_{n_{2}} \cdots \sum_{m_{1}, m_{2}, \ldots}\left|\left\langle f\left|\bar{H}_{1}\right| i\right\rangle\right|^{2} \delta\left(\Delta E+\sum_{i} m_{i} \hbar \omega_{i}\right) \\
& =\frac{2 \pi}{\hbar} J^{2} \sum_{n_{1}, n_{2}, \ldots}\left(1-z_{1}\right) z_{1}^{n_{1}}\left(1-z_{2}\right) z_{2}^{n_{2}} \cdots \sum_{m_{1}, m_{2}, \ldots} \delta\left(\Delta E+\sum_{i} m_{i} \hbar \omega_{i}\right) \prod \exp \left[-\frac{\lambda_{i}}{\hbar \omega_{i}}\right]\left(\frac{\lambda_{i}}{\hbar \omega_{i}}\right)^{m_{i}} \frac{n_{i} !}{\left(n_{i}+m_{i}\right) !}\left[L_{n_{i}}^{m_{i}}\left(\frac{\lambda_{i}}{\hbar \omega_{i}}\right)\right]^{2},
\end{aligned}
$$

where $\Delta E \equiv \bar{E}_{2}-\bar{E}_{1}$. We now use the identity

$$
\sum_{n} \frac{n !\left[L_{n}^{m}(x)\right]^{2} z^{n}}{(n+m) !}=\frac{x^{-m} z^{-\frac{1}{2} m}}{1-z} e^{-2 x \frac{z}{1-z}} I_{m}\left(2 x \frac{\sqrt{z}}{1-z}\right)
$$

to obtain Eq. (3):

$$
\begin{aligned}
k= & \frac{2 \pi}{\hbar} J^{2} \exp \left(-\frac{\Delta E}{2 k_{\mathrm{B}} T}\right) \exp \left[-\sum_{i} \frac{\lambda_{i}}{\hbar \omega_{i}} \frac{1+z_{i}}{1-z_{i}}\right] \sum_{m_{1}, m_{2}, m_{3}, \ldots} I_{m_{1}}\left[2\left(\frac{\lambda_{1}}{\hbar \omega_{1}}\right) \frac{\sqrt{z_{1}}}{1-z_{1}}\right] I_{m_{2}}\left[2\left(\frac{\lambda_{2}}{\hbar \omega_{2}}\right) \frac{\sqrt{z_{2}}}{1-z_{2}}\right] \\
& \times I_{m_{3}}\left[2\left(\frac{\lambda_{3}}{\hbar \omega_{3}}\right) \frac{\sqrt{z_{3}}}{1-z_{3}}\right] \cdots \delta\left(\Delta E+\sum_{i} m_{i} \hbar \omega_{i}\right),
\end{aligned}
$$

where $I_{m_{i}}$ is a modified Bessel function. 
We continue to derive an expression for the transition rate $k$ that allows efficient numerical computation. To this end we move the factor $\exp \left(-\Delta E / 2 k_{\mathrm{B}} T\right)$ in Eq. (A12) inside the sum, where, by virtue of the $\delta$ function, it can be replaced by

$$
\exp \left(-\frac{\Delta E}{2 k_{\mathrm{B}} T}\right) \rightarrow \exp \left(\frac{\sum_{i} m_{i} \hbar \omega_{i}}{2 k_{\mathrm{B}} T}\right)
$$

We furthermore write the $\delta$ function in Eq. (A12) as an integral,

$$
\delta\left(\Delta E+\sum_{i} m_{i} \hbar \omega_{i}\right)=\frac{1}{2 \pi \hbar} \int_{-\infty}^{\infty} d t \exp \left[i\left(\Delta E+\sum_{i} m_{i} \hbar \omega_{i}\right) t / \hbar\right],
$$

and use the Jacobi-Anger identity

$$
\sum_{m=-\infty}^{\infty} I_{m}(x) \exp (i m \theta)=\exp (x \cos \theta)
$$

with $\theta=\omega_{i} t-i \hbar \omega_{i} / 2 k_{\mathrm{B}} T$, to obtain

$$
k=\frac{J^{2}}{\hbar^{2}} \exp \left[-\sum_{i} \frac{\lambda_{i}}{\hbar \omega_{i}} \frac{1+z_{i}}{1-z_{i}}\right] \int_{-\infty}^{\infty} d t \exp \left[i \frac{\Delta E t}{\hbar}+\sum_{i} \frac{\lambda_{i}}{\hbar \omega_{i}}\left(\frac{1+z_{i}}{1-z_{i}} \cos \left(\omega_{i} t\right)+i \sin \left(\omega_{i} t\right)\right)\right] .
$$

We now add a "classical mode" with reorganization energy $\lambda_{\mathrm{cl}}$ and frequency $\omega_{\mathrm{cl}}$ for which $\hbar \omega_{\mathrm{cl}} \ll k_{\mathrm{B}} T$. In Eq. (A16) we replace $\sum_{i}$ by $\sum_{i}^{\prime}$, which now also contains the classical mode. For the classical mode we can make the following approximations:

$$
\cos \left(\omega_{\mathrm{cl}} t\right) \approx 1-\left(\omega_{\mathrm{cl}} t\right)^{2} / 2, \quad \sin \left(\omega_{\mathrm{cl}} t\right) \approx \omega_{\mathrm{cl}} t .
$$

Inserting these approximations into Eq. (A16) leads to

$$
k=\frac{J^{2}}{\hbar^{2}} \exp \left[-\sum_{i} \frac{\lambda_{i}}{\hbar \omega_{i}} \frac{1+z_{i}}{1-z_{i}}\right] \int_{-\infty}^{\infty} d t \exp \left[i \frac{\left(\Delta E+\lambda_{\mathrm{cl}}\right) t}{\hbar}-\frac{\lambda_{\mathrm{cl}} k_{\mathrm{B}} T t^{2}}{\hbar^{2}}+\sum_{i} \frac{\lambda_{i}}{\hbar \omega_{i}}\left(\frac{1+z_{i}}{1-z_{i}} \cos \left(\omega_{i} t\right)+i \sin \left(\omega_{i} t\right)\right)\right]
$$

which is an exact result in the limit $\omega_{\mathrm{cl}} \rightarrow 0$. Due to the addition of the classical mode, which makes sure that the integrand decays to zero for $t \rightarrow \pm \infty$, the integral over time can be numerically performed with high accuracy for all values of $\Delta E$. We note that an arbitrary number of classical modes can be added in this way. The Gaussian factor in the integrand of Eq. (A18) corresponds to the broadening of the $\delta$ function in Eq. (A12) [Eq. (3) in the main text] to a Gaussian, as mentioned in the main text, with a standard deviation $\sqrt{2 \lambda_{\mathrm{cl}} k_{\mathrm{B}} T}$.

\section{APPENDIX B: RELATION AND COMPARISON TO THE MARCUS-LEVICH-JORTNER RATE}

The Marcus-Levich-Jortner (MLJ) formulation [19,20], which goes beyond the semiclassical approximation, assumes an initial state without phonons in the modes that are treated quantum mechanically. Thermal excitations in the classically treated modes are still accounted for. This means that in Eq. (A18) we should take the limit $T \rightarrow 0$ but keep $T$ finite in the second term in the exponent of the integrand. As a result, we obtain

$$
k_{\mathrm{MLJ}}=\frac{J^{2}}{\hbar^{2}} \exp \left[-\sum_{i} \frac{\lambda_{i}}{\hbar \omega_{i}}\right] \int_{-\infty}^{\infty} d t \exp \left[i \frac{\left(\Delta E+\lambda_{\mathrm{cl}}\right) t}{\hbar}-\frac{\lambda_{\mathrm{cl}} k_{\mathrm{B}} T t^{2}}{\hbar^{2}}+\sum_{i} \frac{\lambda_{i}}{\hbar \omega_{i}} \exp \left(i \omega_{i} t\right)\right],
$$

which is an expression that allows more efficient numerical computation than the familiar expression for the MLJ rate:

$$
k_{\mathrm{MLJ}}=\frac{2 \pi}{\hbar} \frac{J^{2}}{\sqrt{4 \pi \lambda_{\mathrm{cl}} k_{\mathrm{B}} T}} \exp \left[-\sum_{i} \frac{\lambda_{i}}{\hbar \omega_{i}}\right] \sum_{m_{1}=0}^{\infty} \sum_{m_{2}=0}^{\infty} \sum_{m_{3}=0}^{\infty} \cdots \frac{\left(\frac{\lambda_{1}}{\hbar \omega_{1}}\right)^{m_{1}}}{m_{1} !} \frac{\left(\frac{\lambda_{2}}{\hbar \omega_{2}}\right)^{m_{2}}}{m_{2} !} \frac{\left(\frac{\lambda_{3}}{\hbar \omega_{3}}\right)^{m_{3}}}{m_{3} !} \cdots \exp \left[-\frac{\left(\Delta E+\lambda_{\mathrm{cl}}+\sum_{i} m_{i} \hbar \omega_{i}\right)^{2}}{4 \lambda_{\mathrm{cl}} k_{\mathrm{B}} T}\right] .
$$

By expanding $\exp \left[\sum_{i} \frac{\lambda_{i}}{\hbar \omega_{i}} \exp \left(i \omega_{i} t\right)\right]$ in a Taylor series and performing the time integral in Eq. (B1) one can show that Eqs. (B1) and (B2) are equivalent.

In Fig. 4 we compare the $\Delta E$ dependence at room temperature of the MLJ rates (B1) (blue curves) with that of the full quantum rates (A18) (red curves) for 20 randomly chosen molecular pairs of the three materials studied in this paper for electrons and holes. As in the main text, we have taken $J=1 \mathrm{meV}$ and $\lambda_{\mathrm{cl}}=0.01 \mathrm{eV}$. It is clear that for $\Delta E<0$ the MLJ rates are, in general, reasonably accurate (yet in some specific cases they are still significantly off) but that they are inapplicable for $\Delta E>0$, where they are 


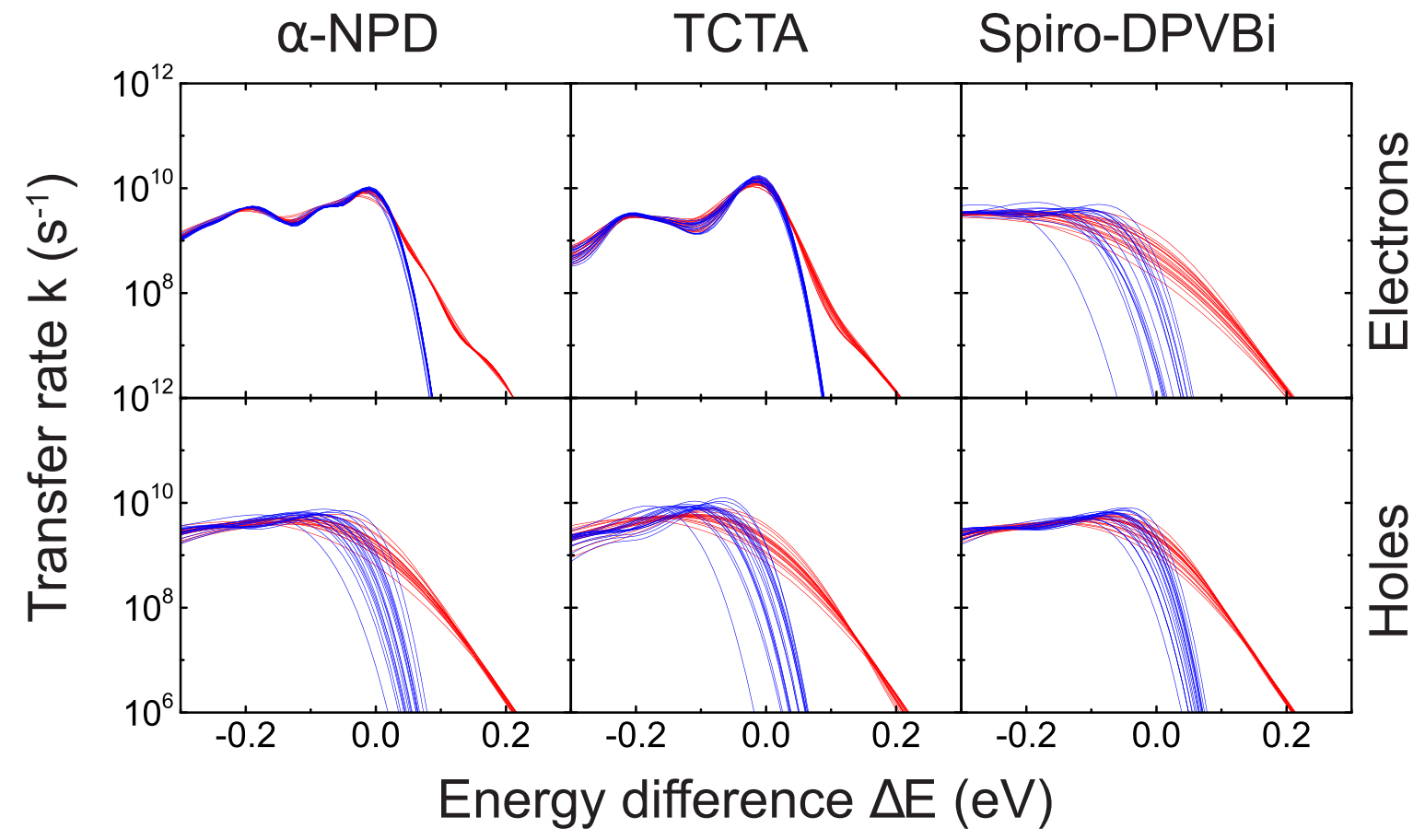

FIG. 4. Full quantum rates (red curves) and MLJ rates (blue curves) for electron and hole transfer at room temperature as a function of $\Delta E$ for 20 randomly chosen pairs in the three materials studied in this work for $J=1 \mathrm{meV}$ and $\lambda_{\mathrm{cl}}=0.01 \mathrm{eV}$.

much too small. In the percolative transport in the disordered amorphous semiconductors studied in this work, the chargecarrier mobility is determined by "difficult but necessary" hops, which will almost always have $\Delta E>0$. Hence, the MLJ rates are inapplicable to modeling charge dynamics in amorphous molecular semiconductors.

\section{APPENDIX C: CALCULATION OF THE INTRAMOLECULAR PHONON FREQUENCIES AND REORGANIZATION ENERGIES}

Morphologies of $\alpha$-NPD, TCTA, and spiro-DPVBi were obtained using the Metropolis Monte Carlo-based simulated annealing protocol DEPOSIT [28], which was applied to simulate the deposition of about 1000 molecules in the vertical direction in simulation boxes of a lateral size of $7 \times 7 \mathrm{~nm}^{2}$ with periodic boundary conditions in the lateral directions. For each material we randomly selected 20 molecules and calculated their intramolecular phonon mode frequencies $\omega_{i}$, the reorganization energies $\lambda_{i}$ associated with all modes $i$, and their total reorganization energies $\lambda$ for charging with an electron or a hole. The calculations were performed using density functional theory (DFT) with a def2-SV(P) [29] basis set and a B3LYP [30] functional as implemented in TURBOMOLE [31]. Every molecule is surrounded by an environment of 100 effective all-electron potentials to take into account the confinement of the molecule by the surrounding molecules [32].

The total reorganization energies $\lambda$ for electrons and holes were calculated using Nelsen's four-point procedure. They are split into charging $\left(\lambda^{0}\right)$ and decharging $\left(\lambda^{*}\right)$ contributions. The results are given in Tables I-III for the three materials. The frequencies $\omega_{i}$ and reduced masses $\mu_{i}$ of the phonon (normal) modes were obtained from the mass-weighted Hessian matrix of force constants. The displacements of all atoms in all three Cartesian directions from their equilibrium positions for the neutral molecule to the equilibrium positions for the

TABLE I. Reorganization energies $\lambda^{0}$ for charging and $\lambda^{*}$ for decharging and the sum $\sum_{i} \lambda_{i}$ of the reorganization energies associated with all intramolecular phonon modes for $20 \alpha$-NPD molecules randomly chosen from a simulated sample of about 1000 molecules. The values indicated with an asterisk $(*)$ were obtained with the linearization method explained in the text.

\begin{tabular}{lccccccc}
\hline \hline \multicolumn{3}{c}{$\alpha$-NPD electron } & & \multicolumn{3}{c}{$\alpha$-NPD hole } \\
\cline { 1 - 4 } \cline { 5 - 7 }$\lambda^{0}(\mathrm{eV})$ & $\lambda^{*}(\mathrm{eV})$ & $\sum_{i} \lambda_{i}(\mathrm{eV})$ & & $\lambda^{0}(\mathrm{eV})$ & $\lambda^{*}(\mathrm{eV})$ & $\sum_{i} \lambda_{i}(\mathrm{eV})$ \\
\hline 0.066 & 0.072 & 0.067 & & 0.116 & 0.104 & 0.108 \\
0.061 & 0.061 & 0.061 & & 0.110 & 0.102 & 0.103 \\
0.058 & 0.057 & 0.059 & & 0.096 & 0.104 & 0.106 \\
0.057 & 0.055 & 0.057 & & 0.154 & 0.157 & 0.170 \\
0.057 & 0.056 & 0.057 & & 0.053 & 0.062 & 0.062 \\
0.061 & 0.060 & 0.061 & & 0.137 & 0.129 & 0.130 \\
0.061 & 0.060 & 0.062 & & 0.159 & 0.135 & 0.188 \\
0.060 & 0.058 & 0.060 & & 0.127 & 0.110 & 0.139 \\
0.060 & 0.060 & 0.061 & & 0.087 & 0.081 & 0.089 \\
0.063 & 0.061 & 0.063 & & 0.067 & 0.060 & 0.067 \\
0.057 & 0.064 & 0.060 & & 0.098 & 0.091 & 0.098 \\
0.057 & 0.055 & 0.057 & & 0.092 & 0.086 & $0.074^{*}$ \\
0.063 & 0.065 & 0.064 & & 0.103 & 0.096 & 0.099 \\
0.062 & 0.064 & 0.063 & & 0.094 & 0.086 & 0.090 \\
0.062 & 0.062 & 0.063 & & 0.106 & 0.108 & 0.105 \\
0.056 & 0.055 & 0.056 & & 0.116 & 0.117 & $0.097^{*}$ \\
0.059 & 0.064 & 0.060 & & 0.071 & 0.066 & 0.071 \\
0.061 & 0.062 & 0.063 & & 0.114 & 0.113 & 0.108 \\
0.055 & 0.058 & 0.056 & & 0.138 & 0.147 & $0.125^{*}$ \\
0.061 & 0.060 & 0.062 & & 0.122 & 0.116 & 0.115 \\
\hline \hline
\end{tabular}


TABLE II. Same as Table I, but for TCTA.

\begin{tabular}{ccccccc}
\hline \hline \multicolumn{3}{c}{ TCTA electron } & & \multicolumn{3}{c}{ TCTA hole } \\
\cline { 1 - 2 } \cline { 5 - 6 }$\lambda^{0}(\mathrm{eV})$ & $\lambda^{*}(\mathrm{eV})$ & $\sum_{i} \lambda_{i}(\mathrm{eV})$ & $\lambda^{0}(\mathrm{eV})$ & $\lambda^{*}(\mathrm{eV})$ & $\sum_{i} \lambda_{i}(\mathrm{eV})$ \\
\hline 0.032 & 0.034 & 0.033 & & 0.017 & 0.017 & 0.016 \\
0.053 & 0.048 & 0.053 & & 0.072 & 0.062 & 0.070 \\
0.033 & 0.035 & 0.033 & & 0.120 & 0.142 & $0.104^{*}$ \\
0.040 & 0.041 & 0.041 & & 0.108 & 0.115 & $0.083^{*}$ \\
0.043 & 0.042 & 0.043 & & 0.083 & 0.086 & $0.071^{*}$ \\
0.037 & 0.043 & 0.038 & & 0.079 & 0.070 & $0.070^{*}$ \\
0.041 & 0.041 & 0.043 & & 0.088 & 0.091 & 0.088 \\
0.039 & 0.038 & 0.039 & & 0.085 & 0.085 & 0.081 \\
0.043 & 0.041 & 0.044 & & 0.149 & 0.111 & $0.082^{*}$ \\
0.031 & 0.033 & 0.032 & & 0.085 & 0.117 & $0.099^{*}$ \\
0.067 & 0.220 & $0.069^{*}$ & & 0.232 & 0.108 & $0.040^{*}$ \\
0.036 & 0.036 & 0.037 & & 0.077 & 0.077 & 0.076 \\
0.028 & 0.031 & 0.029 & & 0.085 & 0.090 & 0.087 \\
0.032 & 0.038 & 0.032 & & 0.085 & 0.091 & 0.092 \\
0.043 & 0.042 & 0.043 & & 0.082 & 0.077 & 0.076 \\
0.034 & 0.035 & 0.035 & & 0.087 & 0.087 & 0.084 \\
0.032 & 0.033 & 0.033 & & 0.113 & 0.141 & $0.147^{*}$ \\
0.027 & 0.030 & 0.028 & 0.083 & 0.077 & 0.077 \\
0.033 & 0.035 & 0.034 & & 0.078 & 0.077 & 0.076 \\
0.037 & 0.038 & 0.039 & 0.080 & 0.078 & 0.077 \\
\hline \hline
\end{tabular}

charged molecule were decomposed in displacements $\Delta q_{i}$ of the phonon modes $i$. The reorganization energies $\lambda_{i}$ were then obtained as $\lambda_{i}=\frac{1}{2} \mu_{i} \omega_{i}^{2} \Delta q_{i}^{2}$.

When the charge-phonon coupling is linear in the displacements $\Delta q_{i}$ of the phonon modes and when these displacements are within the harmonic regime, which are the conditions for applicability of the Hamiltonian (2), we have $\lambda^{0}=\lambda^{*}=$ $\sum_{i} \lambda_{i}$. We see from Tables I-III that this is almost always, to

TABLE III. Same as Table I, but for spiro-DPVBi.

\begin{tabular}{lcccccc}
\hline \hline \multicolumn{3}{c}{ spiro-DPVBi electron } & & \multicolumn{3}{c}{ spiro-DPVBi hole } \\
\cline { 1 - 2 } \cline { 5 - 6 }$\lambda^{0}(\mathrm{eV})$ & $\lambda^{*}(\mathrm{eV})$ & $\sum_{i} \lambda_{i}(\mathrm{eV})$ & & $\lambda^{0}(\mathrm{eV})$ & $\lambda^{*}(\mathrm{eV})$ & $\sum_{i} \lambda_{i}(\mathrm{eV})$ \\
\hline-0.052 & 0.535 & $0.166^{*}$ & & 0.087 & 0.103 & 0.101 \\
0.173 & 0.149 & 0.197 & & 0.133 & 0.198 & 0.149 \\
0.210 & 0.216 & 0.239 & & 0.133 & 0.131 & 0.133 \\
0.151 & 0.150 & $0.136^{*}$ & & 0.089 & 0.091 & 0.099 \\
0.091 & 0.104 & 0.099 & & 0.067 & 0.067 & 0.066 \\
0.138 & 0.139 & 0.142 & & 0.095 & 0.088 & 0.096 \\
0.134 & 0.116 & 0.147 & & 0.067 & 0.069 & 0.068 \\
0.180 & 0.164 & $0.131^{*}$ & & 0.069 & 0.077 & 0.070 \\
0.232 & 0.258 & $0.191^{*}$ & & 0.107 & 0.197 & $0.191^{*}$ \\
0.147 & 0.169 & 0.173 & & 0.103 & 0.099 & 0.106 \\
0.129 & 0.124 & 0.140 & & 0.081 & 0.215 & 0.085 \\
0.171 & 0.183 & 0.189 & & 0.071 & 0.072 & 0.074 \\
0.115 & 0.106 & 0.119 & & 0.064 & 0.070 & 0.067 \\
0.159 & 0.143 & 0.167 & & 0.097 & 0.159 & 0.104 \\
0.088 & 0.105 & 0.101 & & 0.077 & 0.080 & 0.081 \\
0.155 & 0.133 & 0.175 & & 0.081 & 0.098 & 0.090 \\
0.164 & 0.148 & 0.183 & & 0.073 & 0.078 & 0.076 \\
0.102 & 0.105 & 0.105 & & 0.106 & 0.097 & 0.111 \\
0.227 & 0.205 & $0.158^{*}$ & & 0.097 & 0.094 & 0.096 \\
0.094 & 0.099 & 0.098 & & 0.074 & 0.173 & 0.076 \\
\hline \hline
\end{tabular}

a reasonable approximation, the case. In cases where $\sum_{i} \lambda_{i}$ calculated with this method was $30 \%$ larger than $\lambda^{0}$ we used a different method to calculate the $\lambda_{i}$ 's. In those cases, indicated with an asterisk $(*)$ in Tables I-III, we used a linearization method in which we calculated the derivatives $\partial E / \partial q_{i}$ of the total energy of the charged molecule with respect to the phonon mode coordinates $q_{i}$ in the equilibrium structure of the neutral molecule. From these derivatives we calculated $\Delta q_{i}$ as $\Delta q_{i}=$ $\left(\partial E / \partial q_{i}\right) / \mu_{i} \omega_{i}^{2}$ and from these the reorganization energies $\lambda_{i}=\frac{1}{2} \mu_{i} \omega_{i}^{2} \Delta q_{i}^{2}$. In this way we obtain the leading linear term of the charge-phonon coupling. Tables I-III show that this method always yields reasonable values of $\sum_{i} \lambda_{i}$. We note, however, that the method is necessarily approximate because in these cases the displacements are beyond the linear regime of the charge-phonon coupling and/or beyond the harmonic regime. Because these cases are rare, they have no significant effect on the results presented in this paper. We also note that for the first spiro-DPVBi molecule in Table III, $\lambda^{0}$ is negative for charging by an electron. The reason is that the molecule is in a local minimum and can gain energy by crossing a barrier in the direction of the equilibrium structure of the charged molecule. This is accompanied by a very large positive value of $\lambda^{*}$. However, also for this case $\sum_{i} \lambda_{i}$ as calculated with the linearization method has a reasonable value.

\section{APPENDIX D: INFLUENCE OF $\lambda_{\mathrm{cl}}$ ON CHARGE TRANSFER RATES AND MOBILITIES}

Equation (A18) with $\lambda_{\mathrm{cl}}=0.01 \mathrm{eV}$ is used in this work to calculate the full quantum transfer rates, mobilities, and energy
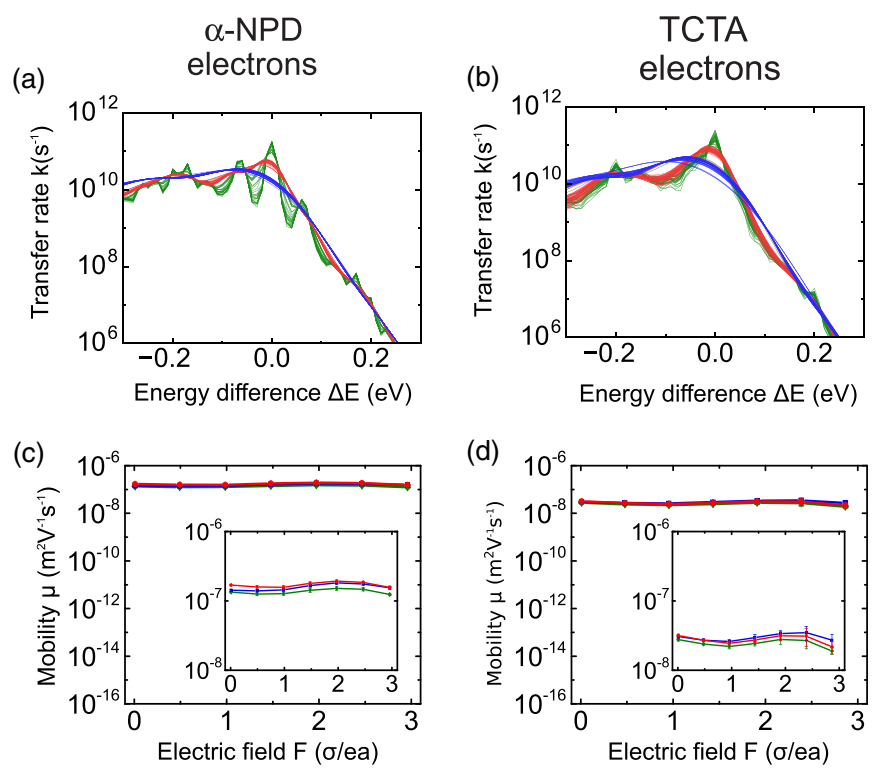

FIG. 5. (a) and (b) Room-temperature full quantum transfer rates as a function of energy difference $\Delta E$ as calculated with Eq. (A18) for electrons in $\alpha$-NPD and TCTA, respectively, for $J=1 \mathrm{meV}$. Green lines: $\lambda_{\mathrm{cl}}=0.001 \mathrm{eV}$. Red lines: $\lambda_{\mathrm{cl}}=0.01 \mathrm{eV}$ [same as in Fig. 2(b)]. Blue lines: $\lambda_{\mathrm{cl}}=0.05 \mathrm{eV}$. (c) and (d) Electron mobilities $\mu$ in $\alpha$ NPD and TCTA, respectively, as a function of electric field $F$ for the different values of $\lambda_{\mathrm{cl}}$ in (a) and (b). The scale of the vertical axis in the main panels is the same as in Fig. 3(a). The insets have a reduced vertical scale to make the differences visible. 
relaxation of electrons and holes in $\alpha$-NPD, TCTA, and spiroDPVBi. We investigate the effect of using different values of $\lambda_{\mathrm{cl}}$ in Fig. 5. Figures 5(a) and 5(b) show the room-temperature rates for electron transfer in $\alpha$-NPD and TCTA, respectively, for $\lambda_{\mathrm{cl}}=0.001 \mathrm{eV}$ (green lines), $0.01 \mathrm{eV}$ [red lines, same results as in Fig. 2(b)], and $0.05 \mathrm{eV}$ (blue lines). Figures 5(c) and 5(d) show the corresponding room-temperature mobilities as a function of electric field. While detailed features in the rates clearly depend on the value of $\lambda_{\mathrm{cl}}$, the variations in the mobilities do not exceed $40 \%$. (Note that $\lambda_{\mathrm{cl}}$ varies by a factor of 50!) This shows that the mobilities are quite insensitive to the choice of $\lambda_{\mathrm{cl}}$. The two considered cases of electrons in $\alpha$-NPD and TCTA are the most critical. For the other cases the mobilities are even less sensitive to the choice of $\lambda_{\mathrm{cl}}$.
[1] H. Bässler, Phys. Status Solidi B 175, 15 (1993).

[2] A. Miller and E. Abrahams, Phys. Rev. 120, 745 (1960).

[3] C. Tanase, E. J. Meijer, P. W. M. Blom, and D. M. de Leeuw, Phys. Rev. Lett. 91, 216601 (2003).

[4] Y. Roichman and N. Tessler, Synth. Met. 135-136, 443 (2003).

[5] S. Baranovskii, O. Rubel, and P. Thomas, Thin Solid Films 487, 2 (2005).

[6] W. F. Pasveer, J. Cottaar, C. Tanase, R. Coehoorn, P. A. Bobbert, P. W. M. Blom, D. M. de Leeuw, and M. A. J. Michels, Phys. Rev. Lett. 94, 206601 (2005).

[7] J. J. Kwiatkowski, J. Nelson, H. Li, J. L. Bredas, W. Wenzel, and C. Lennartz, Phys. Chem. Chem. Phys. 10, 1852 (2008).

[8] V. Rühle, A. Lukyanov, F. May, M. Schrader, T. Vehoff, J. Kirkpatrick, B. Baumeier, and D. Andrienko, J. Chem. Theory Comput. 7, 3335 (2011).

[9] P. Kordt, J. J. M. van der Holst, M. Al Helwi, W. Kowalsky, F. May, A. Badinski, C. Lennartz, and D. Andrienko, Adv. Funct. Mater. 25, 1955 (2015).

[10] P. Friederich, V. Meded, A. Poschlad, T. Neumann, V. Rodin, V. Stehr, F. Symalla, D. Danilov, G. Lüdemann, R. F. Fink, I. Kondov, F. von Wrochem, and W. Wenzel, Adv. Funct. Mater. 26, 5757 (2016).

[11] R. A. Marcus, Rev. Mod. Phys. 65, 599 (1993).

[12] K. Asadi, A. J. Kronemeijer, T. Cramer, L. Jan Anton Koster, P. W. M. Blom, and D. M. de Leeuw, Nat. Commun. 4, 1710 (2013).

[13] K. Vandewal, J. Benduhn, K. S. Schellhammer, T. Vangerven, J. E. Rückert, F. Piersimoni, R. Scholz, O. Zeika, Y. Fan, S. Barlow, D. Neher, S. R. Marder, J. Manca, D. Spoltore, G. Cuniberti, and F. Ortmann, J. Am. Chem. Soc. 139, 1699 (2017).

[14] G. Nan, L. Wang, X. Yang, Z. Shuai, and Y. Zhao, J. Chem. Phys. 130, 024704 (2009).

[15] J. Cottaar, L. J. A. Koster, R. Coehoorn, and P. A. Bobbert, Phys. Rev. Lett. 107, 136601 (2011).
[16] A. Massé, P. Friederich, F. Symalla, F. Liu, V. Meded, R. Coehoorn, W. Wenzel, and P. A. Bobbert, Phys. Rev. B 95, 115204 (2017).

[17] G. D. Mahan, Many-Particle Physics (Plenum, New York, 1990).

[18] R. Egger, C. H. Mak, and U. Weiss, J. Chem. Phys. 100, 2651 (1994).

[19] R. A. Marcus, J. Chem. Phys. 24, 966 (1956).

[20] J. Jortner, J. Chem. Phys. 64, 4860 (1976).

[21] M. Mesta, M. Carvelli, R. J. de Vries, H. van Eersel, J. J. M. van der Holst, M. Schober, M. Furno, B. Lüssem, K. Leo, P. Loebl, R. Coehoorn, and P. A. Bobbert, Nat. Mater. 12, 652 (2013).

[22] A. Massé, P. Friederich, F. Symalla, F. Liu, R. Nitsche, R. Coehoorn, W. Wenzel, and P. A. Bobbert, Phys. Rev. B 93, 195209 (2016)

[23] B. Baumeier, O. Stenzel, C. Poelking, D. Andrienko, and V. Schmidt, Phys. Rev. B 86, 184202 (2012).

[24] P. Friederich, F. Symalla, V. Meded, T. Neumann, and W. Wenzel, J. Chem. Theory Comput. 10, 3720 (2014).

[25] H. van Eersel, R. A. J. Janssen, and M. Kemerink, Adv. Funct. Mater. 22, 2700 (2012).

[26] A. Melianas, F. Etzold, T. J. Savenije, F. Laquai, O. Inganäs, and M. Kemerink, Nat. Commun. 6, 8778 (2015).

[27] M. Mesta, C. Schaefer, J. de Groot, J. Cottaar, R. Coehoorn, and P. A. Bobbert, Phys. Rev. B 88, 174204 (2013).

[28] T. Neumann, D. Danilov, C. Lennartz, and W. Wenzel, J. Comput. Chem. 34, 2716 (2013).

[29] A. Schäfer, H. Horn, and R. Ahlrichs, J. Chem. Phys. 97, 2571 (1992).

[30] A. D. Becke, J. Chem. Phys. 98, 1372 (1993).

[31] R. Ahlrichs, M. Bär, M. Häser, H. Horn, and C. Kölmel, Chem. Phys. Lett. 162, 165 (1989).

[32] M. Dolg, U. Wedig, H. Stoll, and H. Preuss, J. Chem. Phys. 86, 866 (1987). 